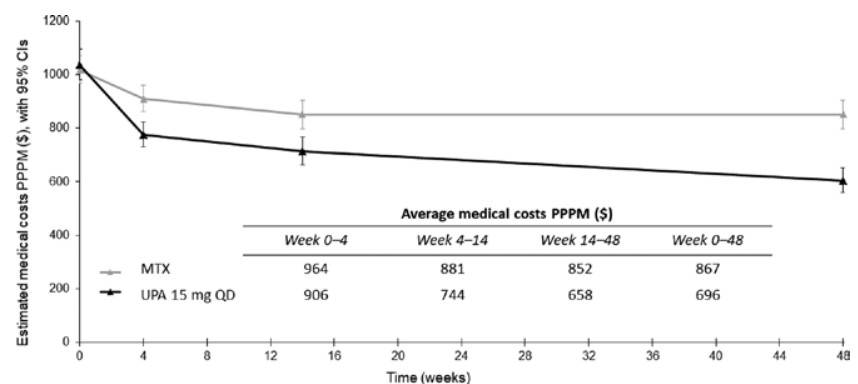

Acknowledgments: Financial support for the study was provided by AbbVie. AbbVie participated in the study design, research, data collection, analysis and interpretation of data, writing, reviewing, and approving the publication. Medical writing services, provided by Joann Hettasch of JK Associates Inc., were funded by AbbVie.

Disclosure of Interests: Arielle Bensimon Employee of: Analysis Group, Min Yang Employee of: Analysis Group, Eli Orvis Employee of: Analysis Group, Jerry Clewell Shareholder of: AbbVie, Employee of: AbbVie, Namita Tundia Shareholder of: AbbVie, Employee of: AbbVie

DOI: 10.1136/annrheumdis-2020-eular.500

\begin{tabular}{l|l}
\hline AB1149 & POOR RESPONSE TO HEPATITIS B VACCINATION IN \\
RHEUMATIC PATIENTS TREATED WITH BIOLOGIC \\
THERAPY - IMPLICATIONS FOR CLINICAL \\
PRACTICE
\end{tabular}

V. C Romão ${ }^{1,2}$, P. Ávila-Ribeiro ${ }^{1,2}$, M. J. Gonçalves ${ }^{1,2}$, A. R. Cruz-Machado, ${ }^{1,2}$, A. Guerreiro ${ }^{2}$, V. Teixeira ${ }^{1,2}$, A. Valido ${ }^{1,2}$, J. Silva-Dinis ${ }^{1,2}$, E. Vieira-Sousa ${ }^{1,2}$, M. J. Saavedra ${ }^{1,2}$, E. Leite $^{3}$, R. Tato Marinho ${ }^{4}$, J. E. Fonseca ${ }^{1,2} .{ }^{1}$ Hospital $^{2}$ Santa Maria, CHULN, Rheumatology Department, Lisbon, Portugal; ${ }^{2}$ Instituto de Medicina Molecular, Faculdade de Medicina, Universidade de Lisboa, Rheumatology Research Unit, Lisbon, Portugal; ${ }^{3}$ Hospital Santa Maria, CHULN, Occupational Medicine Department, Lisbon, Portugal; ${ }^{4}$ Hospital Santa Maria, CHULN, Gastroenterology and Hepatology Department, Lisbon, Portugal

Background: Hepatitis B virus (HBV) vaccination is recommended for rheumatic patients starting biologic therapy. There is some evidence that $\mathrm{HBV}$ vaccination is effective in patients under conventional disease modifying anti-rheumatic drugs (DMARDs), but it is currently unclear whether this also applies to biologics.

Objectives: To assess the efficacy and safety of HBV vaccination in patients with rheumatic diseases treated with biologics.

Methods: We included patients with any inflammatory rheumatic diseases treated with any biologic, who were negative for anti-HBs and anti-HBc and had never been vaccinated for HBV. Engerix $B \otimes$ was administered at 0 , 1 and 6 months and anti-HBs was re-assessed $\geq 1$ month after last dose. Response was defined as anti-HBs $>10 \mathrm{IU} / \mathrm{L}$ and compared against healthy controls $(\mathrm{HC})$ undergoing Occupational Health immunization. Disease flare was evaluated before and until at least 1 month post-vaccination. We recorded serious adverse events (SAE) and immune-related disorders not previously present.

Results: We included 67 patients, most treated with TNF inhibitors (TNFi), and $70 \mathrm{HC}$ (Table 1). Most patients were taking concomitant DMARDs $(69 \%)$ and were in remission/low disease activity $(59 \%)$. Only 20 patients $(30 \%)$ had a positive response to vaccination, in comparison to $68 \mathrm{HC}(97 \%$, $\mathrm{p}<0.001)$. Mean post-vaccination anti-HBs titre was significantly lower in responding patients than $\mathrm{HC}(569 \pm 772$ vs $1316 \pm 811 \mathrm{U} / \mathrm{L}, \mathrm{p}<0.001)$. Responders diagnoses were RA ( $n=8[25 \%])$, PsA ( $n=7$ [39\%]), AS $(n=4$ $[33 \%])$ and IBD-associated SpA $(n=1[100 \%])$. Response was seen in 19/53 patients treated with TNFi $(36 \%)$, but only $1 / 14(7 \%)$ of patients treated with non-TNFi $(p=0.037)$. Importantly, some responders had to temporarily interrupt biologic therapy due to other intercurrences for at least one administration. No clinical or demographic variables were associated with response, including age and disease activity. Fourteen patients (21\%) experienced disease flares, of which 7 were mild and did not require therapy adjustment; 3 patients required minor treatment/dose adjustments; 4 patients had secondary failures that led to switches. There were 3 SAE (acute diverticulitis; abdominal infection; atrial fibrillation and urinary infection) 1-4 months after $1^{\text {st }} / 2^{\text {nd }}$ dose, deemed not to be related to vaccination. One RA patient on infliximab had bilateral uveitis 2 months after the $1^{\text {st }}$ vaccine dose, which resolved with topical therapy.
Table 1. Baseline characteristics of study participants.

\begin{tabular}{lccc}
\hline & Patients (n=67) & Controls ( $\mathrm{n}=70)$ & $\mathrm{p}$ \\
\hline Age (years) & $56 \pm 9$ & $46 \pm 9$ & $<0.001$ \\
Female (\%) & $40(60)$ & $62(89)$ & $<0.001$ \\
Diagnosis (\%) & $32(48)$ & & \\
RA & $18(27) / 13(19)$ & & \\
PsA / AS & $4(6)$ & & \\
Other & $17 \pm 10$ & & \\
Disease duration (years) & $53(79)$ & & \\
Biologic (\%) & $6(9) / 1(1)$ & \\
TNF-inhibitor & $2(3) / 4(6)$ & \\
Tocilizumab / Abatacept & $1(1)$ & \\
Rituximab / Belimumab & $39(58) / 1(1)$ & \\
Anakinra & $6(9) / 3(4)$ & & \\
Conventional DMARDs (\%) & $21(31)$ & \\
MTX / LEF & $29(43) / 5.6 \pm 2.1$ & \\
SSZ / Other & $3.1 \pm 1.4$ & \\
None & $2.2 \pm 1.4$ & \\
Prednisolone (\%) / Dose (mg) & & \\
DAS28 & & \\
ASDAS & & \\
& &
\end{tabular}

Conclusion: In this study, HBV vaccination response was poor and lower in rheumatic patients treated with biologic therapy than in healthy adults. Vaccination was overall safe but there were 4 severe flares and 3 SAE that lead to treatment switch/ interruption, although causal association is difficult to establish. Our data reinforce the recommendation for HBV vaccination prior to starting biologic therapy, possibly even as soon as the diagnosis is established. Alternative HBV vaccination strategies should be investigated in patients already treated with biologics.

Disclosure of Interests: Vasco C Romão: None declared, Pedro Ávila-Ribeiro Grant/research support from: Novartis, Maria João Gonçalves: None declared, Ana Rita Cruz-Machado: None declared, André Guerreiro: None declared, Vítor Teixeira: None declared, Ana Valido: None declared, Joana Silva-Dinis: None declared, Elsa Vieira-Sousa: None declared, Maria João Saavedra: None declared, Ema Leite: None declared, Rui Tato Marinho: None declared, Joao Eurico Fonseca: None declared

DOI: 10.1136/annrheumdis-2020-eular.3966

\section{AB1150 ECONOMIC IMPACT ASSOCIATED TO BIOLOGICAL THERAPY / SYNTHETIC FAME OPTIMIZATION IN A COHORT OF PATIENTS WITH CHRONIC INFLAMMATORY RHEUMATIC DISEASES TREATED BY OBJECTIVES.}

P. Castro $^{1}$, A. Onteniente ${ }^{2} .{ }^{1}$ Glorieta Francisco de las Cabezas, Rheumatology, Madrid, Spain; ${ }^{2}$ Calle del General Aranaz, Pharmacy, Madrid, Spain

Background: Therapeutic decision-making for biologic-therapies/ synthetic FAME (BT/SD) dose optimization, should be based on optimal disease activity results according to a treatment strategy by objectives. The goal of BT optimization is to guarantee long-term effectiveness and safety, maximising economic savings

Objectives: To evaluate BT optimization patterns in patients with rheumatic diseases (RD) and associated economic savings.

Methods: An observational and prospective study, which included a cohort of patients with rheumatoid arthritis (RA), spondyloarthropathies (SA) and psoriatic arthritis (PsA) treated with BT from January 2014 to December 2019. BT optimization, achieved by reducing or prolonging the interval at least one dose, was indicated when patients have more than 6 months of treatment and are in clinical remission (DAS28 $<2.6$ for RA and PSA, and BASDAl $<2$ for $\mathrm{SA}$ ) or minimal clinical activity (DAS28<3.2 for RA and PsA, and BASDAI $<4$ for SA).

Variables were described as frequencies and means. Diagnosis, BT (abatacept, adalimumab, apremilast, baricitinib, certolizumab, etanercept, golimumab, ixekizumab secukinumab, tocilizumab, tofacitinib, and ustekinumab), dose regimens, total treatment duration, time on BT optimization (TO) and treatment costs were collected. Cost savings were calculated per patient by comparing optimization treatment costs to conventional treatment and globally by comparing real cost to theoretical conventional doses cost.

Results: A total of 260 patients were included in the study. Switching were observed in $32.7 \%$. From all patients, $53 \%$ were candidates for BT optimization (according to diagnosis: $60.9 \%$ with RA, followed by $52.2 \%$ with SA and $43.4 \%$ with PsA)

A $40 \%$ of patients with BT optimization were treated with adalimumab and etanercept being also the most common BT used in RD treatment

BT optimization allowed a pharmaceutical saving of $€ \mathbf{1 7 7 , 5 3 9 . 4 0}$ per year against the use of conventional therapy, resulting in a reduction of the total cost of $€ 1, \mathbf{0 6 5}, \mathbf{2 3 6 . 4 0}$ in the last 6 years. The saving per patient / year was $€ 707.63$ for RA; $€ 850,40$ for SA and of $€ 493,21$ for the PsA. 
Conclusion: Therapeutic decision-making based on validated disease activity scales has allowed the BT optimization in approximately $53 \%$ of patients with RD. BT optimization allowed a pharmaceutical saving of $€ 177,539.40$ per year being higher in the SA (€ 850.40) followed by the RA (€ 707.63) and finally the PsA ( $€$ 493.21)

The BT optimization allows to reduce costs maintaining the effectiveness and safety.

Disclosure of Interests: None declared

DOI: 10.1136/annrheumdis-2020-eular.5354

\begin{tabular}{l|l}
\hline AB1151 & COMPLIANCE/CONCORDANCE WITH \\
MYCOPHENOLATE MOFETIL IN PATIENTS WITH \\
CONNECTIVE TISSUE DISORDERS IN COVENTRY.
\end{tabular}

A. Chauhan ${ }^{1}$, N. Lovell ${ }^{1}$, S. Dubey ${ }^{1} .{ }^{1}$ Coventry and Warwickshire, Rheumatology, Coventry, United Kingdom

Background: Connective tissue disorders like Systemic lupus erythematosus (SLE) are multi-organ systemic conditions characterised by disordered immune function. Mycophenolate Mofetil (MMF) is commonly used for treatment of SLE ${ }^{1}$ and other connective tissue disorders like Sjogren's syndrome, myositis and Scleroderma. Compliance with drugs remains a significant issue in management of these conditions and varying reports from across the world ${ }^{2,3}$ continue to show significant lack of concordance resulting in increased disease activity and damage.

Objectives: The aim of this study was to investigate the compliance/concordance specifically with MMF treatment among patients attending clinics at University Hospitals Coventry and Warwickshire NHS Trust (UHCW) with SLE and other connective tissue disorders.

Methods: Ethical approval was obtained through research and development department within the Trust. This is a retrospective study collating non-identifiable hospital pharmacy data in patients who requested the prescription for MMF drug between January 2015 and December2018. Since MMF was required to be prescribed from the hospital (i.e. General practitioners within the region were unable to prescribe it), we have records for all prescriptions for these patients. We extracted information on sample size, frequency of prescription requested and length of follow up. Clinical data were obtained from paper and electronic notes of the patients. Data were analysed using the data analysis tool pack for linear regression, on Microsoft Excel package version 16.29.1.

Results: We recruited 144 patients into this study, (74\%) of these are females. Age range for this group was $2-89$ years, median age was $45( \pm 11.2)$ years with a mean $( \pm S D)$ age of $35.6( \pm 11.2)$ years and a disease duration of $8.8( \pm 6.2)$ years. $73.1 \%$ were White British, the remaining included $8.3 \%$ Indian, 5.5\% Pakistani, 2.7\% Black British, 2\% Caucasian, 2.1\% Chinese, and $6.3 \%$ other. Overall, we had 54 patients with SLE and 90 Patients with other connective tissue disorders. Good compliance (81-100\%) with MMF therapy was seen in 49 patients, (34\%). Poor compliance $(0-20 \%)$ was seen in 13 patients, $(9 \%)$. We found a significant correlation between lack of compliance and risk of flares $(r=0.25, p<0.002)$, displayed in Figure 1. We also found a significant difference in compliance patters depending on diagnosis and also on age. SLE patients were $34 \%$ less compliant with MMF in comparison to other connective tissue disorders. Demographics suggested the degree of compliance increased with age. Patients between 40-69 years of age were $65 \%$ more compliant in comparison to the age $20-39$ years $(p<$ 0.002).

Conclusion: SLE and connective tissue disorder patients within Coventry continue to have issues relating to compliance/concordance with MMF treatment and this appears to be worse in patients with SLE and in the 20-39 years of age. These patients also appear to be getting flares hence, this remains a major problem in the management of these conditions.

References:

[1] Gordon C, Amissah-Arthur MB, Gayed M et al. The British Society for Rheumatology guideline for the management of systemic lupus erythematosus in adults. Rheumatol 2017; doi: 10.1093/rheumatology/ kex286.

[2] Alsowaida N, Alrasheed M, Mayet A, Alsuwaida A and Omair M A. Medication adherence, depression and disease activity among patients with systemic lupus erythematosus. Lupus 2018, 27(2), 327-332. doi:10.1177/0961203317725585.

[3] Chehab G, G M Sauer, J G Richter, R Brinks, R Willers, R FischerBetz, B Winkler-Rohlfing, and M Schneider. Medical Adherence in Patients with Systemic Lupus Erythematosus in Germany: Predictors and Reasons for Non-Adherence - a Cross-Sectional Analysis of the LuLa-Cohort." Lupus (September 2018),1652-60. doi:10.1177/096 1203318785245

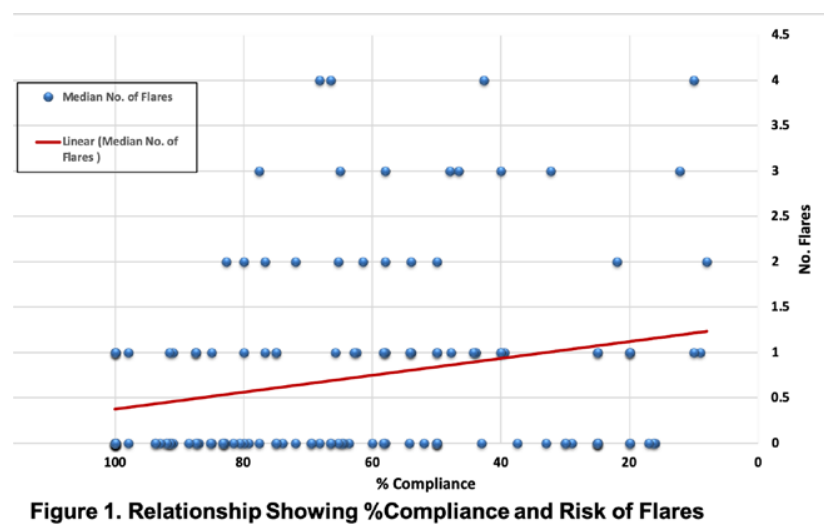

Disclosure of Interests: None declared

DOI: 10.1136/annrheumdis-2020-eular.6415

\section{$\mathrm{AB} 1152$ \\ INCREASED VACCINATION RATE AMONG PATIENTS UNDER BIOLOGICAL DMARDS AFTER INFECTIOUS RISK ASSESSMENT CLINIC}

$\underline{\text { F. Cunha Santos }}^{1}$, A. S. Pinto ${ }^{1}$, S. P. Dinis ${ }^{1}$, C. Quinaz ${ }^{2}$, J. F. Ferreira ${ }^{1,3}$, C. Vaz ${ }^{3}$. ${ }^{1}$ ULS - Unidade de Saúde Local da Guarda, Rheumatology, Guarda, Portugal;

${ }^{2}$ ULS - Unidade de Saúde Local da Guarda, Infectious Diseases, Guarda, Portugal; ${ }^{3}$ Faculdade de Ciências da Saúde, Covilhã, Portugal

Background: Infectious complications are a major concern among immunosuppressed patients. In an attempt to ameliorate these, EULAR released an updated version of their recommendations for vaccinations in patients with rheumatic diseases. These should resonate even further, since our patients are deliberately immunosuppressed in order to achieve remission. In 2018, an Infectious Risk Assessment Clinic (IRAC) was created in our centre to address these issues and prepare patients for biological therapy. This work summarizes our experience.

Objectives: Compare vaccination rate among patients with rheumatic diseases under biological therapy before and after the clinic creation.

Methods: A retrospective observational study was conducted in our Rheumatology Department and IRAC, at Local Health Unit of Guarda. All patients under biological therapy followed in our department from 2010 to 2020 were chart reviewed. Sociodemographic and clinical features were collected: sex, age, rheumatic disease diagnosis, current biological treatment and their vaccination history. Studied vaccines were Pneumococcal 13-valent conjugate, Pneumococcal vaccine polyvalent, Influenza virus, Hepatitis A and B, Herpes Zoster and Tetanus/Diphtheria. Patient was considered vaccinated when the correct schedule was followed by the attending physician. If prescription was outside the stipulated timing, it was considered not vaccinated. Chi-square and Fisher's exact test were used to assess associations and $p<0.05$ was considered statistically significant different.

Results: 65 patients were included, $41(63.10 \%)$ were females, with a mean age of $52.38 \pm 11.11$ years. Diagnosis distribution is the following: rheumatoid arthritis (52.30\%), axial spondylarthritis $(26.20 \%)$, psoriatic arthritis $(12.30 \%)$, peripheral spondylarthritis $(6.20 \%), 1(1.50 \%)$ patient with Sjögren Syndrome and $1(1.50 \%)$ with systemic sclerosis. Most common drugs were anti-TNFa (64.60\%), anti-JAK (15.40\%), anti-IL17A (10.80\%), anti-CD20 (7.70\%) and anti-IL12/IL23 (1.50\%). Of all patients, $24(36.90 \%)$ attended IRAC before starting biological therapy. Table 1 shows prescribed vaccines in both groups and their associations.

Table 1. Prescribed vaccines in both groups $(p<0.05)$.

\begin{tabular}{|c|c|c|c|}
\hline & IRAC attended & IRAC not attended & $\mathrm{P}$ \\
\hline Pneumococcal 13-valent conjugate & & & 0.24 \\
\hline Prescribed & $23(95.83 \%)$ & $34(82.93 \%)$ & \\
\hline Not Prescribed & 1 & 7 & \\
\hline Pneumococcal vaccine polyvalent & & & 0.01 \\
\hline Prescribed & $19(79.17 \%)$ & $19(46.34 \%)$ & \\
\hline Not Prescribed & 5 & 22 & \\
\hline Influenza virus & & & $<0.01$ \\
\hline Prescribed & $22(91.67 \%)$ & $12(29.27 \%)$ & \\
\hline Not Prescribed & 2 & 29 & \\
\hline Hepatitis A & & & 0.05 \\
\hline Prescribed & $3(12.50 \%)$ & $0(0.00 \%)$ & \\
\hline Not Prescribed & 21 & 41 & \\
\hline \multicolumn{4}{|l|}{ Hepatitis B } \\
\hline Prescribed & $12(50.00 \%)$ & $0(0.00 \%)$ & \\
\hline Not Prescribed & 12 & 41 & $<0.01$ \\
\hline Herpes Zoster & & & 0.37 \\
\hline Prescribed & $1(4.17 \%)$ & $0(0.00 \%)$ & \\
\hline Not Prescribed & 23 & 41 & \\
\hline Tetanus/Diphtheria & & & 0.14 \\
\hline Prescribed & $3(12.50 \%)$ & $1(2.44 \%)$ & \\
\hline Not Prescribed & 21 & 40 & \\
\hline
\end{tabular}

